\title{
RELAPSE FOLLOWING GONIOTOMY FOR CONGENITAL GLAUCOMA DUE TO TRABECULAR DYSGENESIS
}

\author{
I. M. RUSSELL-EGGITT ${ }^{1}$, N. S. C. RICE ${ }^{1}$, BARRIE JAY ${ }^{1}$, R. K. H. WYSE ${ }^{2}$ \\ London
}

\begin{abstract}
SUMMARY
Three hundred and thirty-five eyes of 210 patients with congenital glaucoma due to trabecular dysgenesis were treated by goniotomy as the primary procedure in infancy. In 313 eyes $(\mathbf{9 3 . 5 \%})$ glaucoma was controlled at one year following surgery. Using Kaplan Meier actuarial survival analysis we found that eyes controlled in infancy by one or more goniotomies are at risk of relapse of glaucoma for at least 15 years although $93 \%$ of eyes are still controlled five years after the initial surgery. Patients whose symptoms of congenital glaucoma presented at birth were more likely to relapse than those whose symptoms developed in the first few months of life. Eyes requiring multiple goniotomies in infancy were more likely to relapse than those controlled by a single procedure.
\end{abstract}

Goniotomy has remained the primary surgical treatment of choice for buphthalmos since Otto Barkan first applied the technique in 1938. The surgical technique introduced by Arthur Lister at Moorfields Eye Hospital in 1947 and subsequently employed by his successor Noel Rice is essentially as described by Barkan ${ }^{1}$ except that since 1967 the surgery has been performed with the aid of the operating microscope. At least $86 \%$ of eyes with congenital glaucoma due to trabecular dysgenesis are controlled in infancy by a single or by multiple goniotomy operations when the surgery is performed by an experienced surgeon $^{2}$. However the long term results of a large series have not been reported. Although most surgeons advise lifetime follow up the risk of relapse is not known.

The purpose of this study was to analyse the long term course of patients who were controlled for at least one year by goniotomy in infancy.

\section{PATIENTS AND METHODS}

The records of 400 patients with congenital glaucoma of

From: 'Moorfields Eye Hospital, City Road, London. ${ }^{2}$ Institute of Child Health, Guilford Street, London.

Correspondence to: N. S. C. Rice, Moorfields Eye Hospital, City Road, London EC1V 2PD. various aetiologies treated at Moorfields have been analysed using a mainframe Amdahl computer. Information was stored using an SIR database management system with analysis by SAS statistical procedures. Of these 400 , 215 have been identified as having trabecular dysgenesis using Hoskins classification ${ }^{3}$; all their surgery was at Moorfields and there was good documentation of at least one year follow up.

Three hundred and thirty-five eyes of 210 patients with congenital glaucoma due to trabecular dysgenesis were treated by goniotomy as the primary procedure in infancy. In 313 eyes $(93.5 \%)$ the glaucoma was controlled at one year following goniotomy. A single goniotomy was successful in achieving control in $71 \%$ of eyes (239/335) and control was achieved by one or more goniotomies in 93.5\% (313/335). Control at one year was defined as a normal intraocular pressure as determined by applanation tonometry, a stable corneal diameter and stable optic disc appearance. The children were examined regularly under ketamine anaesthesia for corneal clarity and diameter, intraocular pressure and optic disc appearance.

Kaplan Meier actuarial survival curves were plotted to show the proportion of eyes which have not relapsed in patients who are still being reviewed regularly at Moorfields. Kaplan Meier curves demonstrate the overall survival experience of a cohort in a much more informative way than simply calculating the proportion of survivors at the end of a period ${ }^{4}$. The survival curves are corrected for the differing lengths of follow up for each individual, hence the vertical axis is expressed in proportion of patients rather than in numbers (multiply by 100 for percentage of survivors).

Relapse was defined as the development of raised intraocular pressure, increase in optic disc cupping and in older patients, deterioration of the visual fields, leading to further surgery or the institution of medical treatment.

We were interested in estabishing whether relapse had differing prevalence throughout the follow up and to identify the ages when patients should be most carefully 
reviewed. To investigate this further we studied the slopes of the Kaplan Meier survival curves using Hazard function analysis to determine the instantaneous risk of relapse at all times after one year follow up.

In addition to assessing the long term prognosis for all eyes with trabecular dysgenesis controlled by goniotomy we have analysed the cohort in the following ways:

Individual eyes of patients with bilateral trabecular dysgenesis (Fig. 1).

Affected eyes of patients with unilateral trabecular dysgenesis (Fig. 2).

We felt it important to study unilateral cases separately, at least initially, as more aggressive treatment may be given for the better eye of a bilateral case than for the already amblyopic eye of a patient with unilateral glaucoma.

To compare the shape of the Kaplan Meier curve for eyes of patients with bilateral glaucoma with the curve for unilateral disease we used a Log-Rank test which is also calculated by the SAS statistics programme.

Before this study it was our clinical impression that when there was relapse of glaucoma, young children

BILATERAL TRABECULODYSGENESIS

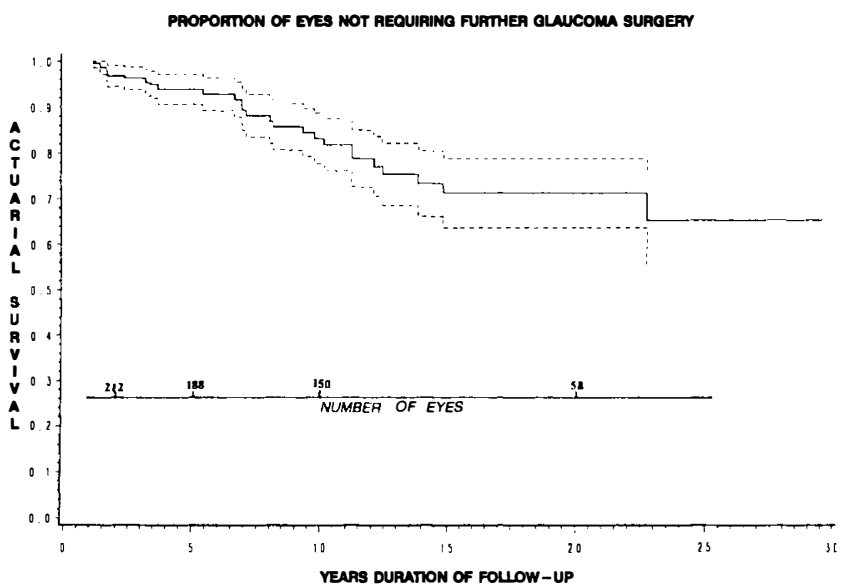

Fig. 1

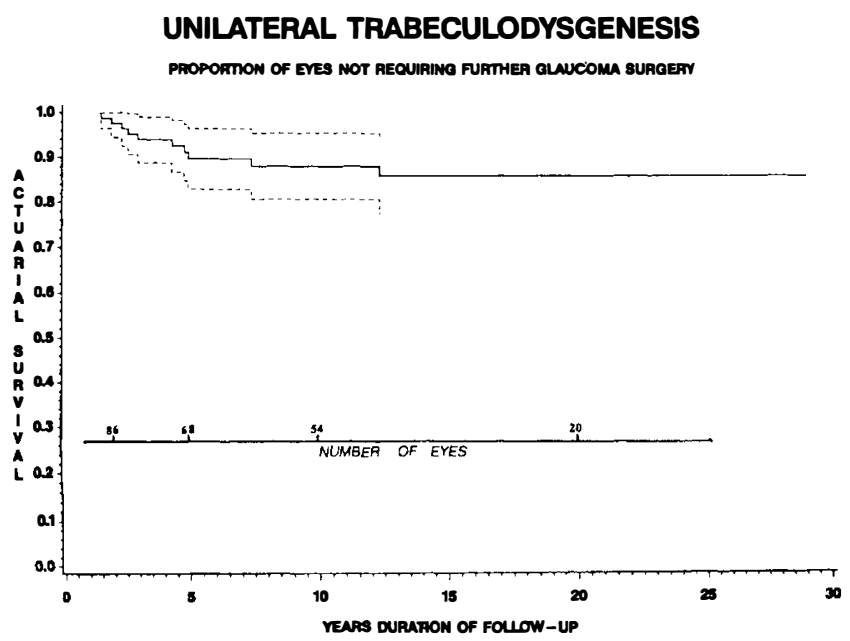

Fig. 2 tended to receive further surgery rather than long-term medical therapy. A further curve was therefore plotted to show the risk to an eye with trabecular dysgenesis requiring further surgery and/or long term medical therapy (Fig. $3)$. This was then compared with the curve defining relapse as requiring further surgery (eyes requiring medication after infancy being counted as survivors) (Fig 4).

\section{RESULTS}

In this series of 210 patients with trabecular dysgenesis there was a male to female ratio of $5: 2$, as compared to $3: 2$ in previous series 5 . Within both the bilateral and unilateral groups the proportion of males to females was also 5:2.

Males with trabecular dysgenesis were equally at risk as females of requiring further surgery after initial control (Chi-Square $\mathrm{P}=0.493$ ).

The age of onset of symptoms such as photophobia, tearing, corneal clouding and enlargement was significantly different in the relapse group. As seen on the bar chart (Fig. 5) 36\% of patients with symptoms at birth had a late relapse, but the proportion was much smaller (15\%) at the commoner age of onset of symptoms between birth

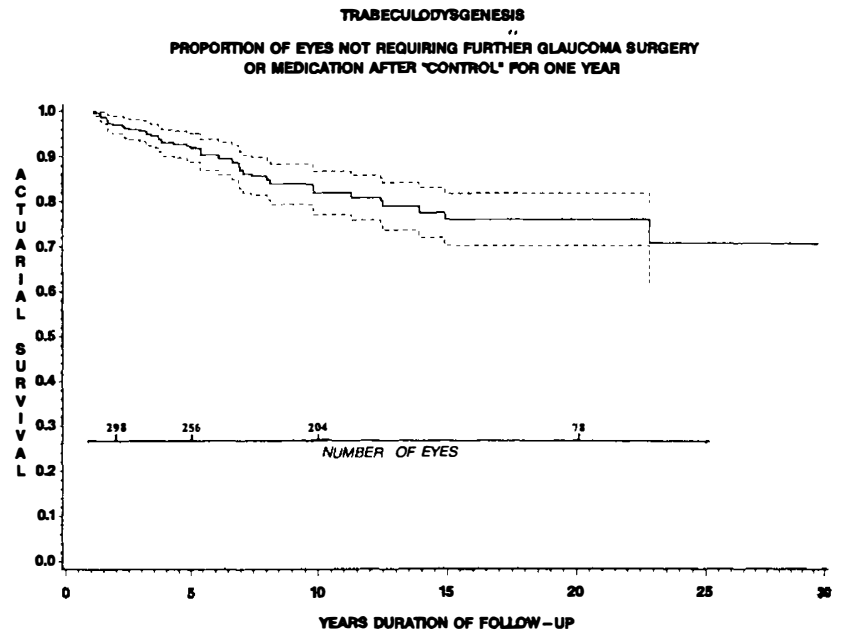

Fig. 3

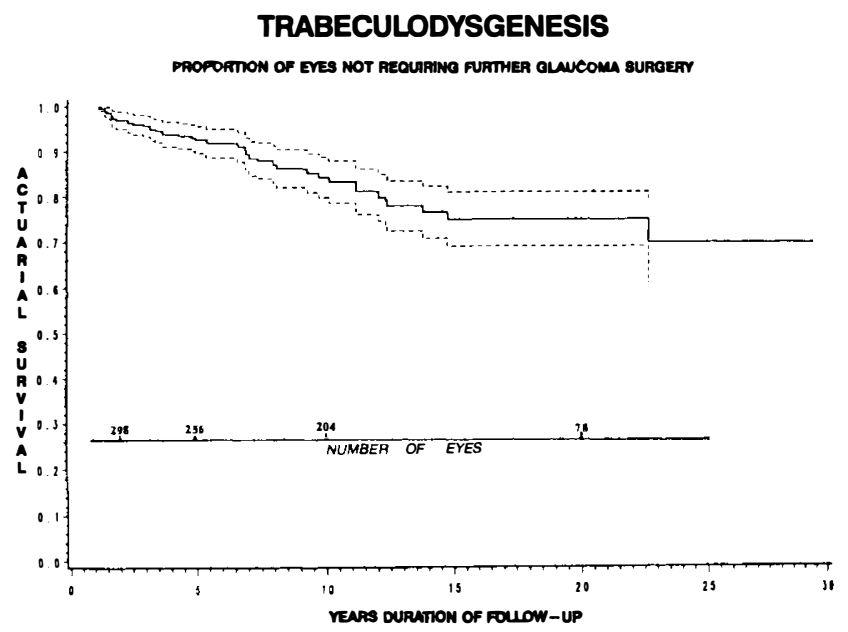

Fig. 4

Figs. 1-4. Kaplan-Meier actuarial survival curve with $95 \%$ confidence limits shown as broken lines. Above the x axis of the curves the number of patients being followed at 2, 5, 10 and 20 years after first surgery is indicated. 


\section{EYES WITH TRABECULODYSGENESIS}

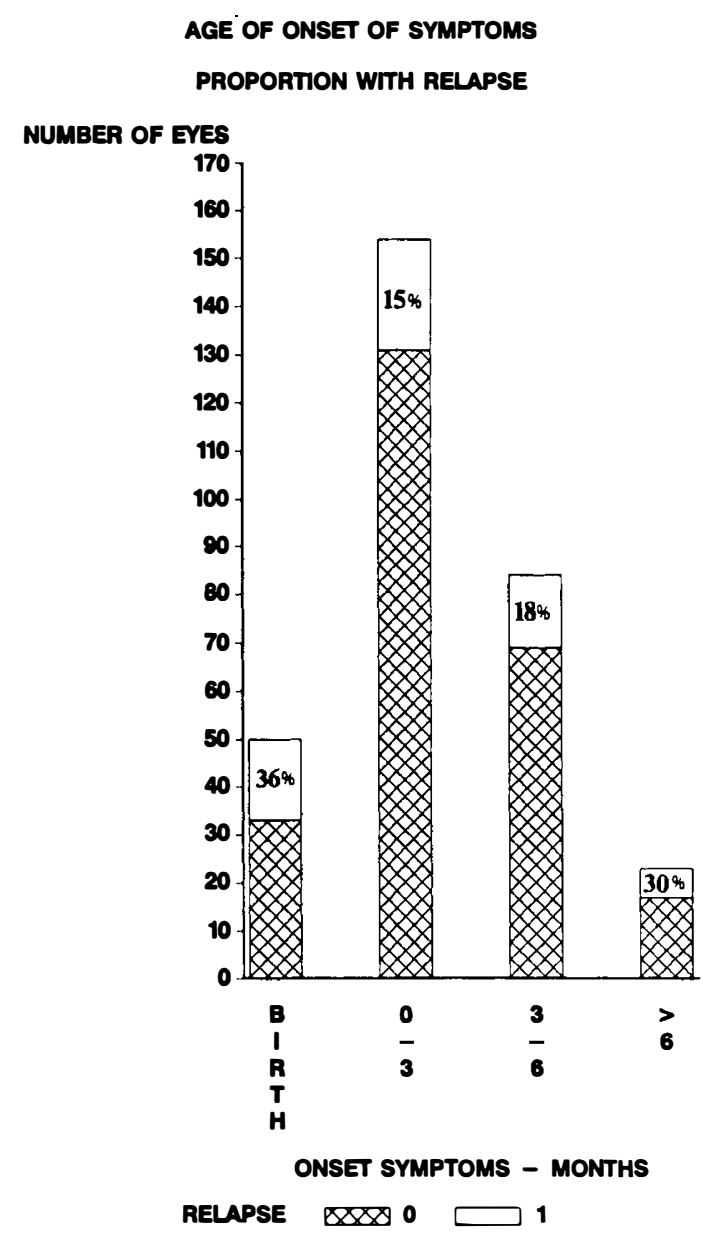

Fig. 5

and three months of age (Chi-Square $P=0.001$, Fisher's 2-Tail $\mathrm{P}=0.002$ ).

Eyes requiring only one goniotomy in infancy were less likely to relapse than those receiving two procedures (ChiSquare $\mathrm{P}=0.018$, Fisher's 2-Tail $\mathrm{P}=0.03$ ) or three goniotomies (Fisher's 2-Tail $P=0.0006$ ) (see Fig. 6).

Relapse occurred in 62 of the 313 eyes in our study. Kaplan Meier actuarial survival curves show the proportion of eyes with trabecular dysgenesis who have not relapsed against the length of follow up.

\section{All Eyes With Trabecular Dysgenesis}

The Kaplan Meier survival curves show the proportion of eyes still controlled without further surgery since infancy. Figure 3 shows that $93 \%$ of eyes, controlled by one or more goniotomies in infancy, remain controlled five years after initial surgery. There are still 256 eyes in our cohort at five years follow up. Hazard function analysis showed that the risk of relapse in previously controlled eyes increases slightly over the first 10 years of follow up but there is no peak age of relapse. Eyes continue to relapse throughout the 30 year follow up, but the number being followed after 10 years makes statistical analysis of attrition rate inappropriate.

Figure 3 is the actuarial survival curve for all eyes except that the definition of relapse includes patients receiving glaucoma medication as well as those receiving further surgery. The slope of the curve appears slightly steeper than in Figure 4, but this does not reach statistical significance.

\section{Eyes of Patients with Bilateral Trabecular Dysgenesis}

Figure 1 is the actuarial survival curve for individual eyes of patients with bilateral trabecular dysgenesis. The Hazard function shows that the risk of relapse, to previously controlled eyes, gradually doubles over the first 12 years of follow up after which the risk of relapse appears to decline, but as previously noted the numbers still followed without any relapse are small.

\section{Patients with Unilateral Trabecular Dysgenesis}

Figure 2 is the actuarial survival curve for glaucomatous individual eyes of patients with unilateral trabecular dysgenesis. Unlike the other survival curves the Hazard function shows a slowly reducing risk of receiving further glaucoma surgery over the first 10 years of follow up.

We compared the survival curves for Figures 1 and 2 using a Log-Rank test and found a greater rate of relapse in the eyes of patients with bilateral as compared with unilateral disease $(\mathrm{p}=0.01)$.

\section{EYES WITH TRABECULODYSGENESIS}

\section{NUMBER OF GONIOTOMIES IN INFANCY \\ PROPORTION WITH LATER RELAPSE}

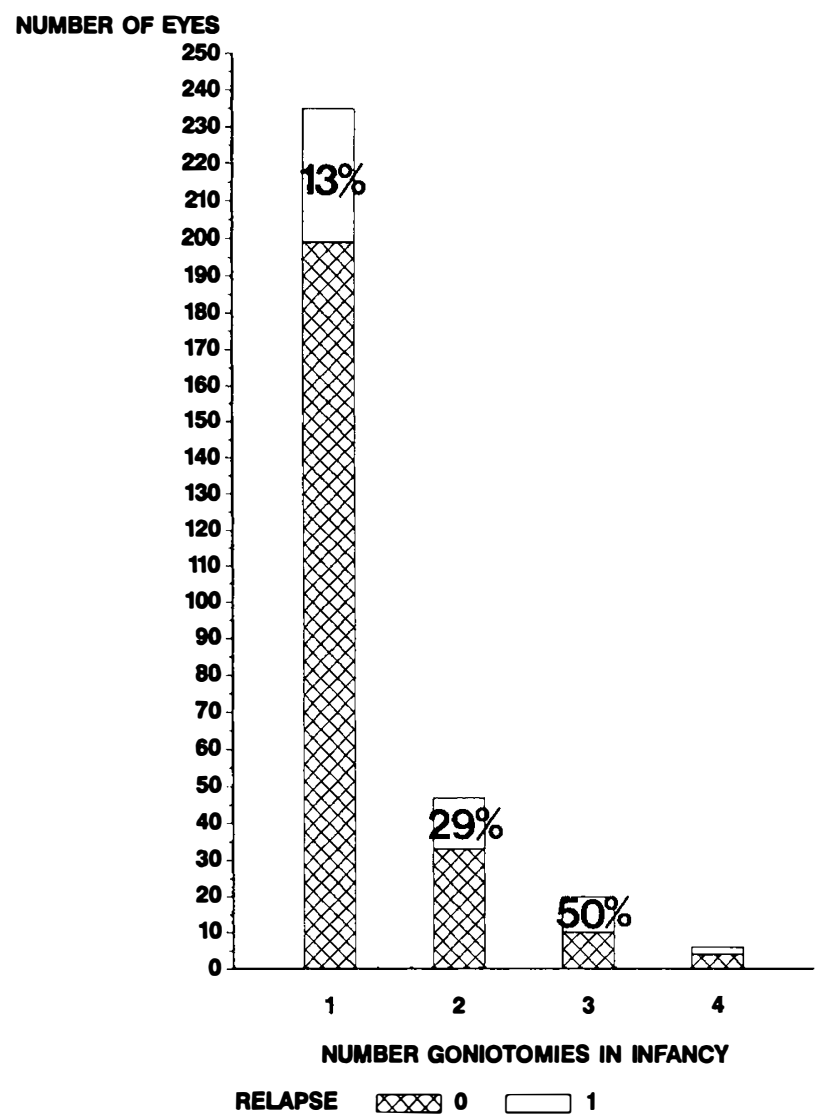

Fig. 6 


\section{DISCUSSION}

Our study analyses the long term results in a cohort of 313 eyes in whom goniotomy was a successful procedure in infancy. Although many studies report the success of goniotomy in children presenting with buphthalmos, the criteria for control vary, an arbitrary intraocular pressure is the most commonly employed. Deluise in 1983 published a review of reports of the initial success of primary goniotomy or trabeculotomy in the management of congenital glaucoma ${ }^{5}$. Various authors since then ${ }^{6-10}$ have reported their series of trabecular dysgenesis including some patients managed by YAG laser goniotomy ${ }^{11}$. Many of the earlier reports included conditions other than trabecular dysgenesis amongst their cases. Assessment of control is multifactorial and intraocular measurement in these eyes is particularly prone to variables, such as depth and type of anaesthesia. Many studies have not quoted the length of patient follow up or if it is relatively short. Therefore we have chosen a more comprehensive definition of glaucoma control for entry into our study.

Our study is of eyes managed by one or more goniotomies for glaucoma due to trabecular dysgenesis. This is our first line procedure and is effective in achieving control in $93.5 \%$ of eyes. Other authors advocate trabeculotomy or trabeculectomy as the primary procedure and have published their short term results. Martin ${ }^{6}$ in 1989 reported $12 / 14$ eyes were controlled with a single trabeculotomy with a follow up of two to three years. No long term study has been reported and it is to be expected that these cases are also prone to relapse with the disadvantage that the conjunctiva has already been disturbed ${ }^{12}$.

Few publications mention the long term prognosis for eyes with trabecular dysgenesis although there is a consensus that follow up should be for life. However, Shaffer ${ }^{10}$ reported $95 \%$ "cure" for eyes with isolated trabecular dysgenesis after one to two goniotomies with no complications or relapse in 82 eyes followed for five to 15 years. He commented that follow-up data was hard to obtain in his population. Our study includes patients already reported by Lister ${ }^{13}$ in 1966 and Rice ${ }^{2}$ in 1977. Rice noted that $6 / 246$ eyes controlled in infancy relapsed at age five to ten years. The proportion of relapsed eyes seems to be low compared to the current paper, but the average follow up of our cases is longer. Barkan ${ }^{1}$ noted that $10 / 152$ eyes with congenital glaucoma, initially controlled, had a relapse of raised intraocular pressure from six months to 13 years after their initial goniotomy. His study probably included eyes other than those with trabecular dysgenesis (such as Axenfeld's syndrome).

This study indicates that although the majority of eyes with congenital glaucoma due to trabecular dysgenesis are initially controlled by goniotomy in infancy, relapse may occur throughout life. Our results also seem to imply that eyes of patients with unilateral disease are less at risk of relapse than individual eyes of bilateral cases. This probably only reflects a less aggressive surgical treatment of these eyes which are often densely amblyopic.

Shaffer demonstrated that eyes with symptoms of con- genital glaucoma at birth require more surgery for initial control, and Barkan observed in 1953 that the long term prognosis of these eyes is worse than those presented in infancy. Our results indicate that the eyes of such patients are also more at risk of later relapse.

\section{CONCLUSIONS}

Our results confirm that goniotomy is a very successful operation for congenital glaucoma due to trabecular dysgenesis in infancy with $93.5 \%$ of eyes having successful control of the glaucoma. There is however a continued risk of those eyes which are initially controlled relapsing later in life. Eyes of children presenting at or soon after birth and those requiring more than one goniotomy, are more at risk of late relapse. The lesson is clear, namely that all patients with congenital glaucoma due to trabecular dysgenesis which is successfully controlled by goniotomy in infancy, should remain under review throughout life.

Key words: Buphthalmos, "Congenital Glaucoma”, Goniotomy, Relapse, "Trabecular dysgenesis"

\section{REFERENCES}

1. Barkan O: Present status on goniotomy. Am J Ophthalmol 1953, 36: 445-53.

2. Rice NSC: The surgical management of the congenital glaucomas. Aust J Ophthalmol 1977, 5: 174.

3. Hoskins HD, Hetherington J, Shaffer RN, Welling AM: Developmental glaucomas: diagnosis and classification, In: Symposium on glaucoma, Transactions of New Orleans Academy of Ophthalmology. St Louis, CV Mosby, 1975; Chapter 10: 194-197.

4. Grunkemeier GL and Starr A: Actuarial analysis of surgical results: Rationale and method. Ann Thoracic Surg 1977, 24: 404-8.

5. DeLuise VP and Anderson DR: Primary infantile glaucoma (congenital glaucoma). Surv Ophthalmol 1983,28:1-19.

6. Martin BB: External trabeculotomy in the surgical treatment of congenital glaucoma. Aust N Z J Ophthalmol 1989, 17: 299-301.

7. McGinnity FG, Page AB, Bryars JH: Primary congenital glaucoma: twenty years experience. Ir J Med Sci 1987, 156: 364-5.

8. Quaranta CA, Rossi Brunori P, Vigasio F, Gatta GP, Semeraro F, Quaranta L: [Goniotomy in congenital glaucoma: therapeutic results] Bull Soc Ophthalmol Fr 1989, 89: 1061-7.

9. Catalano RA, King RA, Calhoun JH, Sargent RA: One versus two simultaneous goniotomies as the initial surgical procedure for primary infantile glaucoma. $J$ Pediatr Ophthalmol Strabis 1989, 26: 9-13.

10. Shaffer RN: Prognosis in primary infantile glaucoma (Trabeculodysgenesis) In Krieglstein GK, Leydhecker W eds. Glaucoma update II Berlin: Springer-Verlag 1983: $185-188$

11. Senft SH, Tomey KF, Traverso CE: Neodymium-YAG laser goniotomy vs surgical goniotomy. A preliminary study in paired eyes. Arch Ophthalmol 1989, 107: 1773-6.

12. Miller MH and Rice NSC. Trabeculectomy combined with $\beta$ radiation for congenital glaucoma. Br J Ophthalmol 1991, 75: (In press).

13. Lister A: The prognosis in congenital glaucoma. Trans Ophthalmol Soc UK 1966, 86: 5-18. 